\title{
Article \\ Adaptive Integral Sliding Mode based Path Following Control of Unmanned Surface Vehicle
}

\author{
José Antonio González-Prieto ${ }^{1, *(\mathbb{D})}$, Carlos Pérez-Collazo ${ }^{1 *(1)}$ and Yogang Singh ${ }^{2}$ (1) \\ 1 Defense University Center at the Spanish Naval Academy, Plaza de España, s/n, Marín 36920, Galicia, \\ Spain \\ 2 Industrial Systems Engineering (ISyE)-FlandersMake@Ghent University, Graaf Karel de Goedelaan 2B, \\ Geb.A, Kortrijk 8500 , Belgium. \\ * Correspondence: jose.gonzalez@cud.uvigo.es
}

\begin{abstract}
This paper investigates the path following control problem for a unmanned surface vehicle (USV) in the presence of unknown disturbances and system uncertainties. The simulation study combines two different types of sliding mode surface based control approaches due to its precise tracking and robustness against disturbances and uncertainty. Firstly, an adaptive linear sliding mode surface algorithm is applied, to keep the yaw error within the desired boundaries and then an adaptive integral non-linear sliding mode surface is explored to keep an account of the sliding mode condition. Additionally, a method to reconfigure the input parameters in order to keep settling time, yaw rate restriction and desired precision within boundary conditions is presented. The main strengths of proposed approach is simplicity, robustness with respect to external disturbances and high adaptability to static and dynamics reference courses without the need of parameter reconfiguration.
\end{abstract}

Keywords: Unmanned Surface Vehicle; Guidance, Navigation and Control; Path Following; Adaptive Sliding Mode

\section{Introduction}

With the growing advancement in the sensor technology and navigation aids, USVs are becoming a popular tool in maritime domain for several applications ranging from environmental monitoring, military surveillance to scientific surveying and data collection. Mission oriented approach of USVs subject them to several types of maritime environment comprising of wind, wave and sea surface currents leading to requirement of designing and developing several autonomy levels for successful operation. Henceforth, design and development of approaches for Guidance, Navigation and Control (GNC) of a USV is an important research area for constructing operational and tactical approaches for seven different operational autonomy level of USVs as described by International Maritime Organisation (IMO).

Guidance and control of USV plays an important role in motion control system to manipulate the forces to enable a USV to follow a desired path whilst maintaining the stability. Three approaches, namely, waypoint control, path following control and trajectory tracking are generally considered in the domain of marine robotics to enable a USV to follow a designated path [1,2]:

- Waypoint control: In this strategy, Line of Sight (LOS) based approach is adopted to follow a certain waypoints, generated heuristically, in the required maritime environment.

- Path following control: In this strategy, a path generated through path planning algorithms is used as a reference, to be followed with no temporal constraints. Here, USV should converge and follow the desired path without any time constraints and simultaneously satisfies its assigned velocity profile. 
- Trajectory tracking: In this strategy, temporal constraints are enforced upon the path generated using path planners. This is predominantly used with fully actuated marine vehicles reasoned with better manoeuvring capabilities.

\subsection{State of the Art}

The problem of path following control is highly non-linear in nature and has been studied from a perspective of observed disturbance control using sliding mode control (SMC) approach. The SMC problem for USVs, subjected to, higher order non linear operational disturbances, have been studied with varying control approaches like sliding mode [4-7]; fuzzy sliding mode [9]; proportional derivative fuzzy [10]; backstepping [11-14]; backstepping with adaptive radial basis function neural network [15]; sine function-based non-linear feedback [16]; hyperbolic tangent based nonlinear control [17]; sigmoid based nonlinear control [18]; function adaptive neural path following control [19]; model predictive control [20,21]; and non-linear feedback power functions [22].

In order to make control robust to disturbances and uncertainties, several approaches has been proposed in the SMC literature, see [23-33]. Some proposals of advanced sliding manifolds include recursive nonlinear sliding manifolds [34,35], non linear full order dynamics [36,37], sliding surfaces with adaptive damping parameters [38-40] and, in the last years, a vast collection of homogeneity based works, see [41] for instance. Applications of the properties of homogeneous systems is an important field of study in the current development of analysis and design of nonlinear controllers and observers. Homogeneity simplifies analysis and design of nonlinear control systems since the homogeneous vector fields have many properties similar to linear one and provides solutions with finite-time and fixed-time stability.

The dynamics generated by an homogeneous controller can be seen as a lineal dynamic system with an adaptive gain that grows to $\infty$ as $|x(t)| \rightarrow 0$, generating the well know singularity at the origin which is undesired for real applications. Nevertheless, as commented in [42], the practical implementation of homogeneous dynamics system designed in the continuous time domain prevents the use of explicit Euler discretization scheme to achieve a mere copy of the continuous time approach due to its simplicity. This type of discretization is considered inappropriate, especially when set-valued functions has to be considering, causing numerical chattering and sensitivity to the gains. As a result, without addressing the discretization issue, any comparison between homogeneous based solutions and other types of proposals may potentially lead to unfair conclusions

Based on the aforementioned results, in order to keep the discretization process simple, an adaptive lineal sliding mode surface law, that includes a nested integral sliding surface is introduced in this work. In this case, the dynamics flows with adaptive and finite damper gain, avoiding the effects of the peaking transient response inherent to linear systems and allowing fast responses at steady state, approximating the behaviour obtained with homogeneous solutions.

\subsection{Major Contributions}

The paper makes following contributions to the current state of existing approaches to SMC techniques for USVs:

- The proposed adaptive control approach is reconfigurable, without parametric adjustment, for various input trajectories and environmental disturbances of maritime environment, as it is shown through various simulation studies conducted in the manuscript.

- Because of the low-pass filtering properties related to the second order adaptive linear dynamics generated at the sliding variable, the cross over frequency $\left(\omega_{c}\right)$ of the system response can be used in the algorithm to estimate bound of the disturbance derivative. This implies that frequencies over $\omega_{c}$ does not affect the performance of the sliding variable response. From a practical implementation 


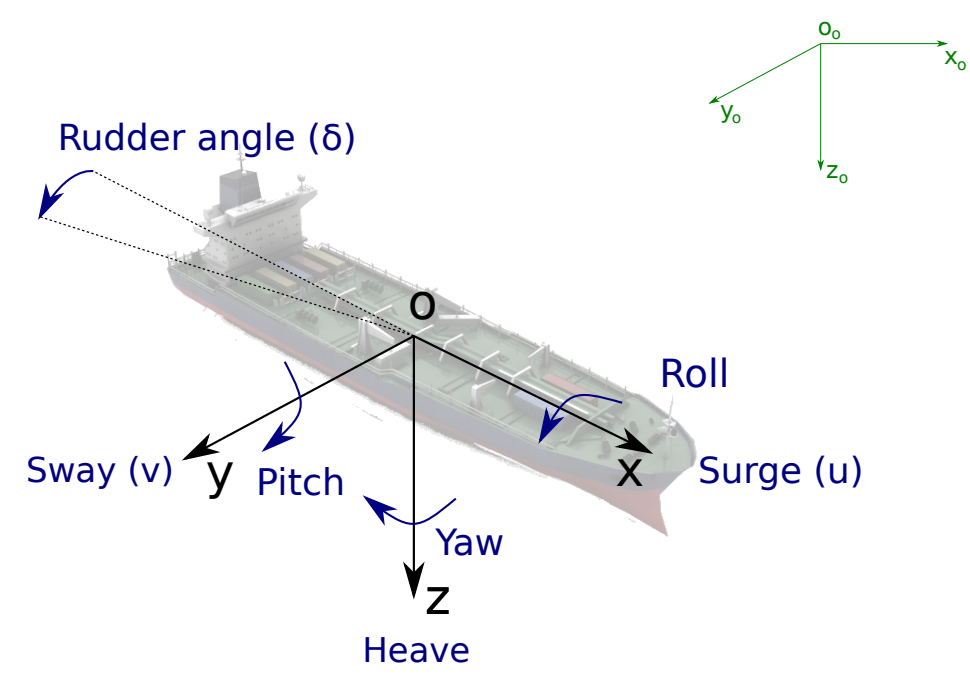

Figure 1. 6 DOF motion representation with North-East-Down coordinate system (green) and body fixed reference frame (black).

perspective, this feature brings some advantages if the estimation of the maximum value of the disturbance derivative is a difficult task to solve.

- The proposed adaptive profile generates a low/high gain variation that depend on the absolute value of the error. This allows to avoid the saturation of the control input when the error is large (gain is small) and to create responses at steady state (gain is large) that generates fast disturbance compensation.

- The use of an scheme based on the adaptive placement of two poles related to a second order dynamical system with critical damped, allows to generate fast overdamped responses that avoids the introduction of considerable overshoots.

This paper has been structured as follows. First, in Section 2 we present the nonlinear dynamic model of the path following problem, the desired objectives to be achieved and a theoretical stability tool that is used in the posterior analysis of the control algorithm. Then, Section 3 describes the proposed adaptive integral sliding mode (AISM) algorithm. Results from numerical simulations are then presented and discussed in Section 4. Finally, conclusions are drawn in Section 5.

\section{Problem Statement}

The motion of the USV is shown in Figure 1, where a six degrees of freedom (DOF) model is presented. The earth fixed Oo is an inertial reference frame fixed to the earth's surface and the body fixed with origin $\mathbf{O}$ is a moving coordinate frame that it is fixed to the craft as in given in [1]. It is assumed an homogeneous mass distributed and xz-plane symmetrical, such that origin of the body fixed reference frame is chosen to be coincident with the center of the gravity. If we consider the path following problem the dynamics of heave, roll, and pitch can be neglected, so that the reduced model dynamics are given as

$$
\begin{aligned}
m\left(\dot{u}-v r-x_{c} r^{2}\right) & =X \text { (Surge) } \\
m\left(\dot{v}+u r+x_{c} \dot{r}\right) & =Y \text { (Sway) } \\
I_{z} \dot{r}+m x_{c}(\dot{v}+u r) & =N \text { (Yaw) }
\end{aligned}
$$

where $\mathrm{m}$ is the mass, $\mathrm{u}$ is the surge velocity, $\mathrm{v}$ the sway velocity, $\mathrm{r}$ the yaw rate, $I_{z}$ the rotational inertia with respect to $\mathrm{z}$ axis, $x_{c}$ is the $\mathrm{x}$ coordinate of the vehicle center in the fixed body reference frame and $\mathrm{X}, \mathrm{Y}$ and $\mathrm{N}$ are the external forces and moments with respect to the surge, sway and yaw, respectively. 
Assumption of constant forward speed and using the ship's Norrbin nonlinear mathematical model, see [43], implies that the steering equations of motion can be obtained as

$$
\begin{aligned}
\dot{\psi}(t) & =r(t) \\
\dot{r}(t) & =f(r)+g \delta(t)+d(t)
\end{aligned}
$$

where, $\psi(t)$ is the yaw (orientation) angle, $r(t)$ is the yaw rate, $\delta(t)$ is the rudder angle (the control variable to be designed) and $d(t)$ is an unknown term to be compensated that includes parametric uncertainty and external disturbances (wind, waves, mobile loads). The dynamics functions are given as

$$
\begin{aligned}
g & =-\frac{K}{T} \\
f(r) & =-\frac{K}{T} H(r) \\
H(r) & =a_{1} r+a_{2} r^{3}
\end{aligned}
$$

where $(K, T)$ are hydrodynamic coefficients and $\left(a_{1}, a_{2}\right)$ are Norrbin coefficients.

In the path following problem it is required that the yaw angle $\psi$ follows a reference angle $\psi_{r}$ by means of the design of the rudder control signal $\delta(t)$. The following assumptions are taking account in this work.

Assumption 1. $d(t)$ in (4) satisfies the following restriction

$$
|\dot{d}(t)| \leq \dot{d}_{\max }
$$

with $\dot{d}_{\text {max }}>0$ a positive real number.

Assumption 2. Henceforth, it is assumed that a reference yaw establish the desired input to be tracked, which can be obtained by means of path planning algorithms, that account for different environment constraints as in [44-47].

A dynamic reference model is used, in this work, to generate the desired course $\left(\psi_{r}(t), \dot{\psi}_{r}(t), \ddot{\psi}_{r}(t)\right)$.

The objective is to design a control law that creates overdamped responses with minimal overshooting (undershooting) and robustness properties for response of the yaw error, which is defined as

$$
e(t)=\psi(t)-\psi_{r}(t)
$$

In order to check the control performance of the proposed controller for the path following problem, we consider the following performance analysis indices mentioned in $[12,14]$.

$$
\begin{aligned}
M A E & =\frac{1}{t_{\infty}-t_{0}} \int_{t_{0}}^{\infty}|e(t)| d t \\
M I A & =\frac{1}{t_{\infty}-t_{0}} \int_{t_{0}}^{\infty}|\delta(t)| d t \\
M T V & =\frac{1}{t_{\infty}-t_{0}} \int_{t_{0}}^{\infty}|\delta(t)-\delta(t-\tau)| d t
\end{aligned}
$$

where $\tau$ is the sampling time used in the simulation.

Furthermore, to check out the robustness properties of the solution, we compare the results with the algorithms proposed in $[12,14]$ applying the following conditions:

- $\quad$ As in $[12,14]$, we test two problems that uses two different types of reference input signals: step and sinusoidal. 
- $\quad$ The tests includes results without disturbances $(d(t)=0)$ and with disturbances $(d(t) \neq 0)$.

- The algorithm parameters are configured in the case of the step input reference without disturbances, such that all solutions provide the same value of the MIA index at the end of the test time.

- After that, the algorithms parameters are fixed and tested in the case of step with disturbances and in the case of the sinusoidal input reference. In this way we check the robustness of the solutions with respect to its capacity of adaptation to different scenarios from a specific parameter configuration.

The following theorem is introduced in order to analyse the stability properties of the AISM proposed solution.

Theorem 1. Consider the following cascade system

$$
\begin{aligned}
& \dot{z}_{1}=f_{1}\left(t, z_{1}\right)+g_{1}\left(t, z_{1}, z_{2}\right) z_{2} \\
& \dot{z}_{2}=f_{2}\left(t, z_{2}\right)
\end{aligned}
$$

where $z_{1} \in \mathbb{R}^{n}, z_{2} \in \mathbb{R}^{m}, f_{1}\left(t, z_{1}\right)$ is continuously differentiable in $\left(t, z_{1}\right)$, and $f_{2}\left(t, z_{1}\right)$ and $g_{1}\left(t, z_{1}, z_{2}\right)$ are continuous and locally Lipschitz in $z_{2}$ and $\left(z_{1}, z_{2}\right)$, respectively.

The dynamics of (10) when $z_{2}=0$ are

$$
\dot{z}_{1}=f_{1}\left(t, z_{1}\right)
$$

If systems (12) and (11) are globally uniformly asymptotically stable (GUAS) and we know a $C^{1}$ Lyapunov function $V\left(t, z_{1}\right)$, two class- $K_{\infty}$ functions $\phi_{1}$ and $\phi_{2}$, a class- $K \phi_{3}$ function and a positive semidefinite function $W\left(z_{1}\right)$ such that

$$
\begin{aligned}
\phi_{1}\left(\left\|z_{1}\right\|\right) & \leq V\left(t, z_{1}\right) \leq \phi_{2}\left(\left\|z_{1}\right\|\right) \\
\frac{\partial V}{\partial t}+\frac{\partial V}{\partial z_{1}} f_{1}\left(t, z_{1}\right) & \leq-W\left(z_{1}\right) \\
\left\|\frac{\partial V}{\partial z_{1}}\right\| & \leq \phi_{3}
\end{aligned}
$$

Besides, for each fixed $z_{2}$ there exists a continuous function $\zeta: \mathbb{R}^{+} \rightarrow \mathbb{R}^{+}$such that

$$
\begin{gathered}
\lim _{s \rightarrow \infty} \zeta(s)=0 \\
\left\|\frac{\partial V}{\partial z_{1}} g_{1}\left(t, z_{1}, z_{2}\right)\right\| \leq \zeta\left(\left\|z_{1}\right\|\right) W\left(z_{1}\right)
\end{gathered}
$$

Then we can conclude that the cascade system (10) and (11) is GUAS.

Proof. See [48] .

\section{Adaptive Integral Sliding Mode Surface Control Design}

Derivation of $e(t)$ in (6) leads to

$$
\dot{e}(t)=r(t)-\dot{\psi}_{r}(t)
$$

An adaptive sliding surface $s(t)$ variable is defined as

$$
s(t)=\dot{e}(t)+\lambda(e) e(t)
$$

with $\lambda(e)$ a real positive time varying parameter. 
Consider the integral term $\bar{s}(t)$

$$
\bar{s}(t)=\int_{0}^{t} s(t) d t
$$

Let's choose the control law as

$$
\delta(t)=\frac{1}{g}\left(-f(r)+\ddot{\psi}_{r}(t)-\lambda(e) \dot{e}(t)-\dot{\lambda}(e) e(t)-\alpha(s, \bar{s}) s(t)-\gamma(e) \bar{s}(t)\right)
$$

with $\lambda(e)$ defined as

$$
\lambda(e)=\max \left(\lambda_{\min }, \lambda_{\max }-\left(\frac{\lambda_{\max }-\lambda_{\min }}{|e(0)|}\right)|e(t)|\right)
$$

, the variable $z(t)$, related a new sliding surface, defined as

$$
z(t)=s(t)+\frac{\alpha}{2} \bar{s}(t)
$$

and with the parameters $\alpha(s, \bar{s}), \gamma(\alpha)$ and $\delta(e)$ given as

$$
\begin{aligned}
\dot{\alpha}(s, \bar{s}) & =\kappa|z|^{\delta} \operatorname{sign}(z) \operatorname{sign}(s) \\
\gamma(\alpha) & =\frac{\alpha^{2}}{4} \\
\delta(e) & =\left(\frac{\delta_{\text {max }}-\delta_{\text {min }}}{|e(0)|}\right)|e(t)|+\delta_{\text {min }}
\end{aligned}
$$

Derivation of $\gamma(\alpha)$ and $\lambda(e)$ are given as

$$
\begin{aligned}
& \dot{\gamma}(\alpha)=\frac{\alpha}{2} \dot{\alpha} \\
& \dot{\lambda}(e)=\left\{\begin{array}{cl}
-\left(\frac{\lambda_{\max }-\lambda_{\min }}{|e(0)|}\right) \operatorname{sign}(e(t)) \dot{e}(t) & \text { if } \lambda>\lambda_{\text {min }} \\
0 & \text { if } \lambda \leq \lambda_{\text {min }}
\end{array}\right.
\end{aligned}
$$

The control algorithm is designed by an appropriate selection of the parameters $\lambda_{\text {max }}, \lambda_{\text {min }}, \alpha(0), \kappa, \delta_{\text {max }}$ and $\delta_{\text {min }}$, as it will be introduced in the numerical simulations section.

Theorem 2. Consider the ship course dynamics described in (4) that complies with assumption 1. The application of the control law (21) to dynamic system (4) implies that the closed compact set $\Omega_{e}$ defined as

$$
\Omega_{e}=\left\{(e(t), \dot{e}(t)) \in \mathbb{R}^{2}:|e(t)|<\frac{\mu}{|\cos (\theta)||\sin (\vartheta)|} \wedge|\dot{e}(t)|<\frac{\mu}{|\cos (\theta)||\cos (\vartheta)|}\right\}
$$

is GUAS with $\mu, \theta$ and $\vartheta$ given as

$$
\begin{aligned}
& \mu=\sqrt[\delta+1]{\frac{\dot{d}_{\text {max }}}{\kappa}} \\
& \theta=\operatorname{atan}(\lambda) \\
& \vartheta=\operatorname{atan}\left(\frac{\lambda}{2}\right)
\end{aligned}
$$


Proof. Application of control law (21) to dynamic system (4) creates the following cascade system.

$$
\begin{aligned}
& \dot{e}(t)=-\lambda e(t)+s(t) \\
& \dot{s}(t)=-\alpha s(t)-\gamma \bar{s}(t)+d(t)
\end{aligned}
$$

The dynamics of $\dot{e}(t)$ when $s(t)=0$ (dynamics of the yaw error at the sliding condition) are

$$
\dot{e}(t)=-\lambda e(t)
$$

with $\lambda>0$. Therefore system (35) is GUAS, with exponential convergence.

Derivation of $\dot{s}(t)$ leads to

$$
\ddot{s}(t)+\alpha \dot{s}(t)+\gamma s(t)+\dot{\alpha} s(t)+\dot{\gamma} \bar{s}(t)+\dot{d}(t)=0
$$

From (25), (27) and (23) it is obtained

$$
\ddot{s}(t)+\alpha \dot{s}(t)+\frac{\alpha^{2}}{4} s(t)+\dot{\alpha} z(t)+\dot{d}(t)=0
$$

Substitution of $\dot{\alpha}$ from (24) implies that the second order dynamics equation related to $s(t)$ is

$$
\ddot{s}(t)+\alpha \dot{s}(t)+\frac{\alpha^{2}}{4} s(t)+\kappa|z|^{\delta+1} \operatorname{sign}(s)+\dot{d}(t)=0
$$

Applying assumption $1,|z|>\sqrt[\delta+1]{\frac{\dot{d}_{\max }}{\kappa}}$ implies that

$$
\kappa|z|^{\delta+1} \operatorname{sign}(s)+\dot{d}=\rho_{z} s
$$

with $\rho_{z}>0$. Therefore the characteristic polynomial of (36) is Hurwitz for all $z(t) \notin \Omega_{z}$ where

$$
\Omega_{z}=\{z(t) \in \mathbb{R}:|z(t)|<\mu\}
$$

with $\mu$ defined in (30).

This implies that (34) is GUAS with respect to the closed set $\Omega_{z}$. Note that dynamics in (36) can be viewed as a second order linear dynamics with adaptive critical damping (exponential convergence related to the fastest response with no overshooting), being perturbed by the overestimation $\rho_{z} s$ caused by the compensation of the unknown term.

Inside $\Omega_{z}$ we have that

$$
\left|s(t)+\frac{\alpha}{2} \bar{s}(t)\right|<\mu
$$

which geometrically entails:

$$
\begin{aligned}
& |\bar{s}(t)|<\frac{\mu}{|\sin (\vartheta)|} \\
& |s(t)|<\frac{\mu}{|\cos (\vartheta)|}
\end{aligned}
$$

with $\vartheta$ defined in (32).

Inside $\Omega_{S}$ we have that

$$
|\dot{e}(t)+\lambda e(t)|<\frac{\mu}{|\cos (\vartheta)|}
$$


Following the previous approach implies that:

$$
\begin{aligned}
& |e(t)|<\frac{\mu}{|\cos (\vartheta)||\sin (\theta)|} \\
& |\dot{e}(t)|<\frac{\mu}{|\cos (\vartheta)||\cos (\theta)|}
\end{aligned}
$$

with $\theta$ defined in (31).

Applying Theorem 1 with

$$
\begin{aligned}
\phi_{1}(\|e\|) & =k_{1} e^{2} \\
\phi_{2}(\|e\|) & =k_{2} e^{2} \\
\phi_{3}(\|e\|) & =k_{3}|e| \\
W(e) & =k_{4} e^{2} \\
V(e) & =\frac{1}{2} e^{2} \\
\zeta(e) & =\frac{k_{5}}{k_{4}|e|}
\end{aligned}
$$

where $k_{1}<0.5, k_{2}>0.5, k_{3}>1.0, k_{4}<\lambda$ and $k_{5}>1.0$, entails that cascade system given in (33) and (34) is GUAS with respect to the closed compact sets $\Omega_{e}$ and $\Omega_{s}$, respectively.

Let's note that

- $\quad$ The size of $\Omega_{e}, \Omega_{s}$ and $\Omega_{z}$ depend on adaptive values of $\lambda, \alpha$ and $\delta$.

- $|z(t)| \rightarrow 0$ implies that $\dot{\alpha} \rightarrow 0$, so the value of $\alpha$ is stabilised at the steady-state of $z(t)$, that is, inside $\Omega_{z}$.

\section{Numerical simulations}

In this section we introduce numerical simulations of the path following problem with parameters given in Table 1 and being executed under the following assumption.

Assumption 3. The numerical simulations are executed using the explicit Euler method with fixed sampling time $\tau=0.1 \mathrm{~s}$.

\begin{tabular}{lr}
\hline Parameter & Value \\
\hline$K$ & 0.21 \\
$T$ & 107.76 \\
$a_{1}$ & 13.17 \\
$a_{2}$ & 16323.46
\end{tabular}

Table 1: Model parameters.

\subsection{Constant yaw reference}

This test is presented in [14] with a required a change in the yaw orientation angle from zero initial condition up to 50 degrees assuming that $d(t)=0$. Table 2 show the parameters used in [14]. Based on this results the parameter $a_{2}$ of the synergetic controller

\begin{tabular}{lr}
\hline Parameter & Value \\
\hline$k_{1}$ & 0.0017 \\
$\omega$ & 0.6000
\end{tabular}

Table 2: Nonlinear concise backstepping controller parameters. 
presented in [12] is changed to achieve the same MIA at the end of the simulation. Table 3 show the parameters used with this algorithm.

\begin{tabular}{lr}
\hline Parameter & Value \\
\hline$a_{1}$ & 0.090 \\
$a_{2}$ & $\mathbf{1 . 8 9 1}$ \\
$T_{1}$ & 28.000
\end{tabular}

Table 3: Synergetic controller parameters.

The parameters of the AISM algorithm are obtained as follows

- Consider a settling time $t_{s}=150 \mathrm{~s}$, a maximum desired yaw rate $r_{\max }=\frac{0.70 \pi}{180}$ degrees per second and a required precision $\epsilon=1.0 e-3$.

- The value of $\alpha(0)$ is obtained assuming an exponential convergence of the error from initial condition $e(0)$ to desired precision $\epsilon$ with a desired settling time $t_{s}$

$$
\alpha(0)=\frac{-\log \left(\frac{\epsilon}{|e(0)|}\right)}{t_{s}}=0.0451
$$

- The value of $\lambda_{\text {min }}$ is related to the initial conditions of the problem and the maximum desired yaw rate as

$$
\lambda_{\min }=\frac{r_{\max }}{|e(0)|}=0.014
$$

and $\lambda_{\max }$ is calculated as

$$
\lambda_{\max }=2.0 \lambda_{\min }=0.028
$$

- $\quad$ The value of $\kappa$ must be higher than $\dot{d}_{\text {max }}$ in order to obtain a small value for $\mu$. Because of the low-pass filtering properties of (36), the value of $\dot{d}_{\text {max }}$ can be further refined by estimating the cross over frequency $\omega_{\mathcal{c}}(t)$ of the second order system related to $s(t)$

$$
\omega_{\mathcal{c}}(t)=\frac{2}{\alpha(t)}
$$

Therefore $\kappa$ is calculated as an adaptive gain that takes account of $\omega_{\mathcal{C}}$ and the desired precision

$$
\kappa=\frac{\omega_{c}(t)}{\epsilon}
$$

- The values of $\delta_{\min }$ and $\delta_{\max }$ are related by means of the condition $\delta_{\max }=2.0 \delta_{\min }$. The value of $\delta_{\min }$ is adjusted with simulations such that the value of the performance index MIA is equal, at the end of test time, to the value obtained with benchmark selected controllers. This choice leads to the following numerical values of $\delta_{\min }$ and $\delta_{\max }$

$$
\begin{aligned}
& \delta_{\text {min }}=1.76952 \\
& \delta_{\max }=3.53904
\end{aligned}
$$

This condition generates an adequate adaption of the value of $\delta$ that allows to obtain the desired low / high gain profile with respect to the absolute value of $e(t)$.

States and control effort are provided in Figure 2 where it is observed that all the solutions provide a similar setting time. Although the evolution of the yaw error is 

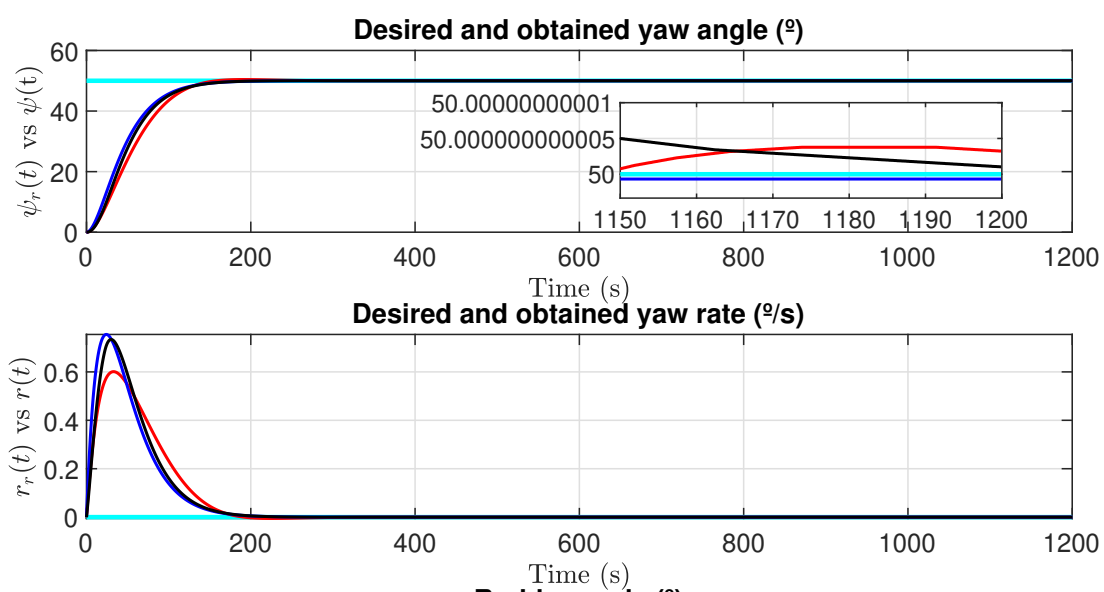

Rudder angle ( $\left.{ }^{\circ}\right)$

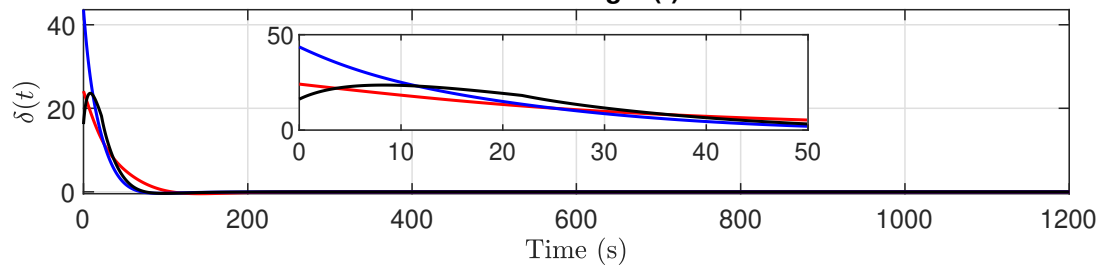

Figure 2. Constant yaw reference test with $d(t)=0$. States and control. Cyan line: Reference ; Red line: Concise backstepping (Zhang et al.); Blue line: Synergetic (Muhammad et al.); Black line: Adaptive sliding mode (González-Prieto et al.)

similar, it can be observer in the detail of the control effort that the evolution of the rudder angle is quite different compared with previous algorithms.

Time evolution of performance indices are shown in Figure 3, with a detailed view of MIA performance index at the end of the test, and final numerical values in Table 4. Figure 4 shows the evolution of the adaptive parameters used at the proposed AISM algorithm.

\begin{tabular}{lrrr}
\hline Algorithm & MAE & MIA & MTV \\
\hline Concise Backstepping [14] & 0.042227 & 0.011348 & $3.6396 \mathrm{e}-5$ \\
Synergetic [12] & 0.035641 & 0.011348 & $6.4436 \mathrm{e}-5$ \\
AISM & 0.038557 & 0.011348 & $4.6133 \mathrm{e}-5$
\end{tabular}

Table 4: Constant yaw reference test with $d(t)=0$. Performance indices.

Next, in order to test the robustness of the algorithms, the following disturbance is considered in (4)

$$
\begin{aligned}
d(t) & =D\left[\cos \left(\omega_{d} t\right)+0.83 \sin \left(3.29 \omega_{d} t-0.14\right)\right. \\
& +1.23 \cos \left(8.12 \omega_{d} t+0.26\right) \\
& \left.+0.65 \sin \left(1.37 \omega_{d} t+0.36\right) e^{\cos \left(2.21 \omega_{d} t+0.13\right)}\right]
\end{aligned}
$$

with

$$
\begin{aligned}
D & =0.0025 \\
\omega_{d} & =0.0703 \frac{\mathrm{rad}}{\mathrm{s}}
\end{aligned}
$$




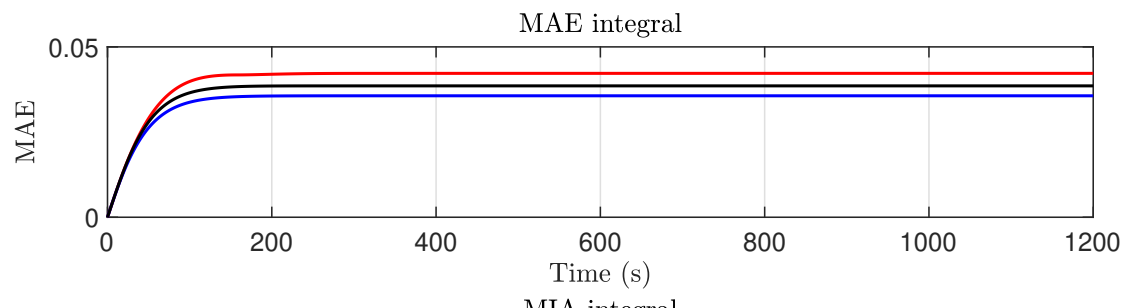

MIA integral
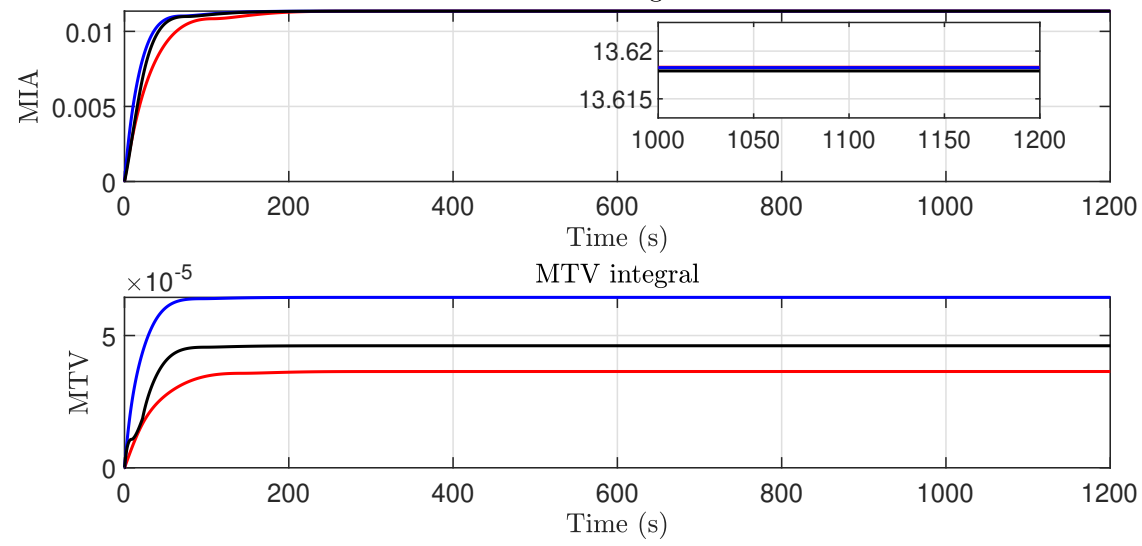

Figure 3. Constant yaw reference test with $d(t)=0$. Performance indices evolution.Cyan line : Reference ; Red line: Concise backstepping (Zhang et al.); Blue line: Synergetic (Muhammad et al.); Black line: Adaptive sliding mode (González-Prieto et al.)

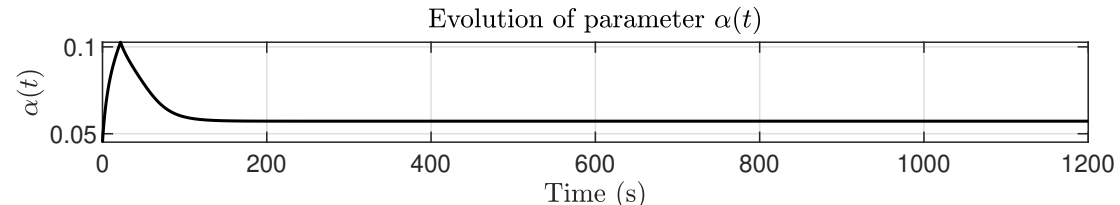

Evolution of parameter $\lambda(t)$

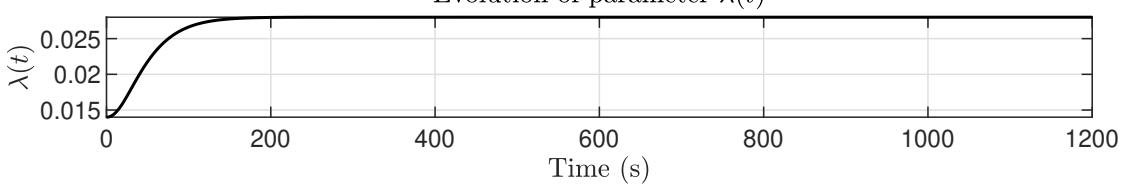

Evolution of parameter $\delta(t)$
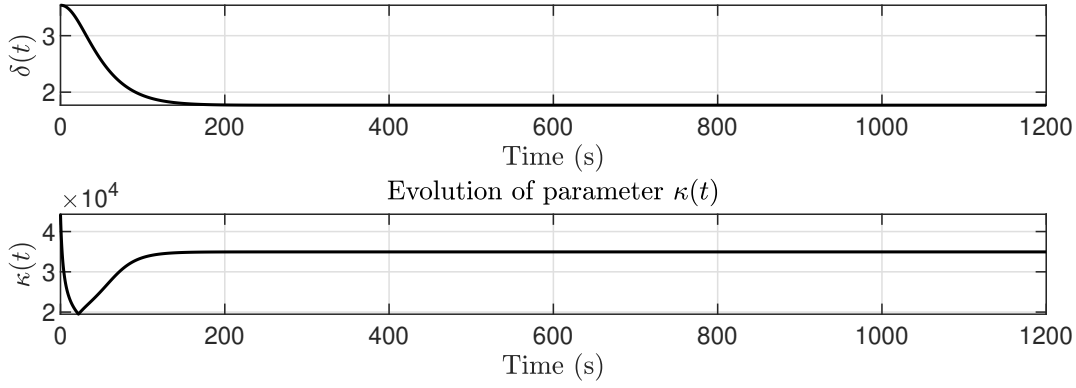

Figure 4. Constant yaw reference test with $d(t)=0$. Adaptive parameters evolution. 

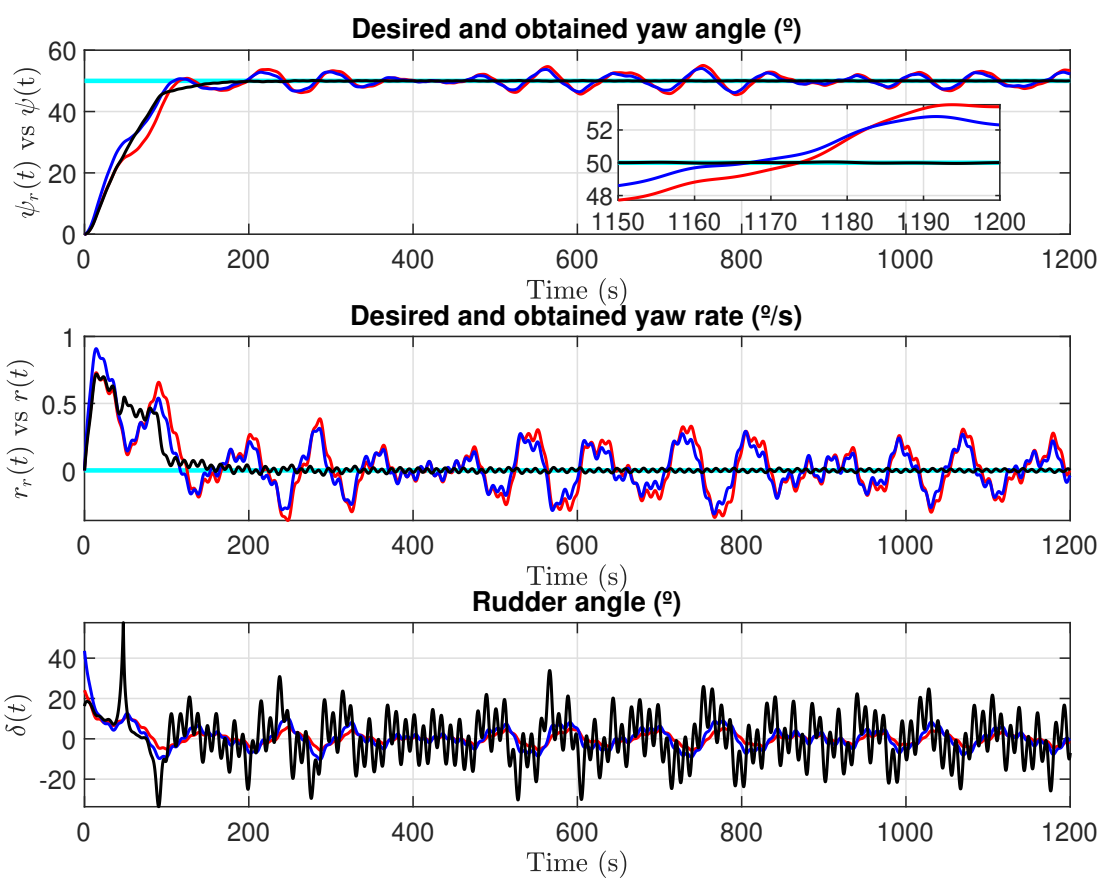

Figure 5. Constant yaw reference test with $d(t) \neq 0$. States and control.Cyan line : Reference ; Red line: Concise backstepping (Zhang et al.); Blue line: Synergetic (Muhammad et al.); Black line: Adaptive sliding mode (González-Prieto et al.)

States and control effort are provided in Figure 5 where it is clear that the proposed AISM cancels the effect of the external disturbance, keeping the desired performance at steady-state generating a rudder angle control that provides fast response attenuation without generation of overshooting. Figure 6 shows the evolution of the sliding variable $s(t)$ and the external disturbance $d(t)$ introduced to test robustness properties of the compared algorithms.

\subsection{Sinusoidal yaw reference}

In this case, as in [14], the yaw reference to follow is a sinusoidal signal defined as

$$
\omega_{d}=\frac{50 \pi}{180.0} \sin \left(\frac{2 \pi}{600.0}\right)
$$

where the initial yaw angle is

$$
\psi(0)=\frac{10 \pi}{180.0}
$$

States and control effort are provided in Figure 7 where it is clear that AISM is capable to follow the yaw reference with no appreciable delay keeping the desired settling time. As in the previous test, results with sinusoidal reference are tested introducing disturbance (50). Figure 8 shows the states and control effort obtained in this case, where, as in the constant reference test, the steady-state performance and the settling time obtained with AISM are preserved despite the presence of the external unknown disturbance.

\section{Conclusions and future works}

In this work we have proposed an approach to develop an adaptive integral sliding mode procedure to design a nonlinear controller for the path following of surface 

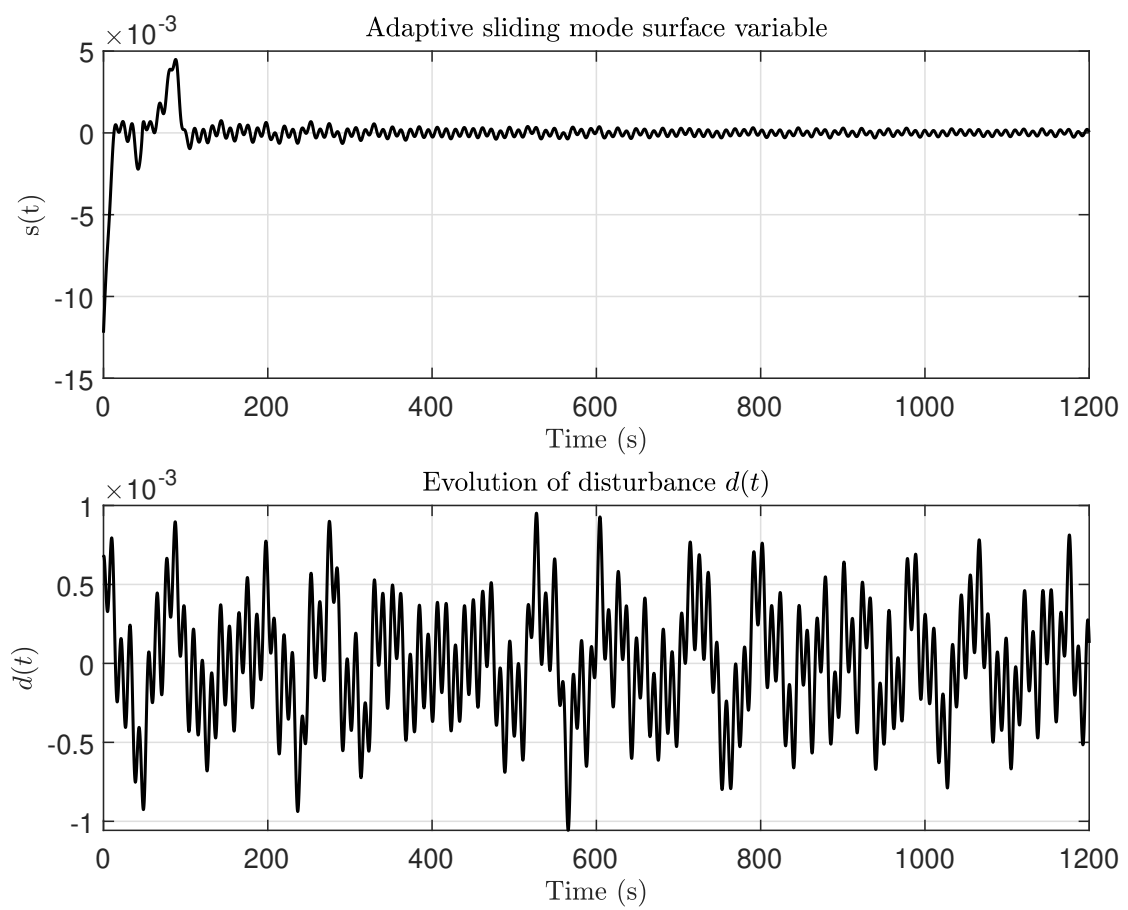

Figure 6. Constant yaw reference test with $d(t) \neq 0$. Sliding mode variable $s(t)$ and external disturbance $d(t)$.
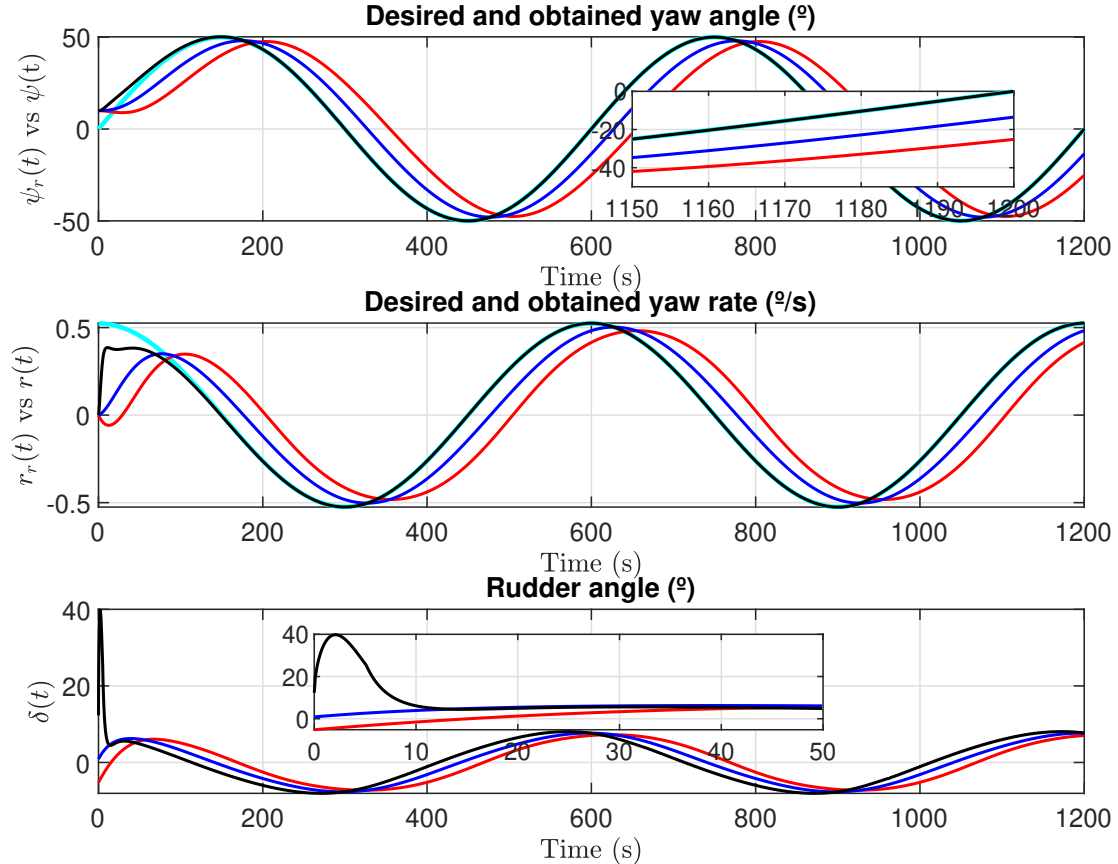

Figure 7. Sinusoidal yaw reference test with $d(t)=0$. States and control.Cyan line : Reference ; Red line: Concise backstepping (Zhang et al.); Blue line: Synergetic (Muhammad et al.); Black line: Adaptive sliding mode (González-Prieto et al.) 

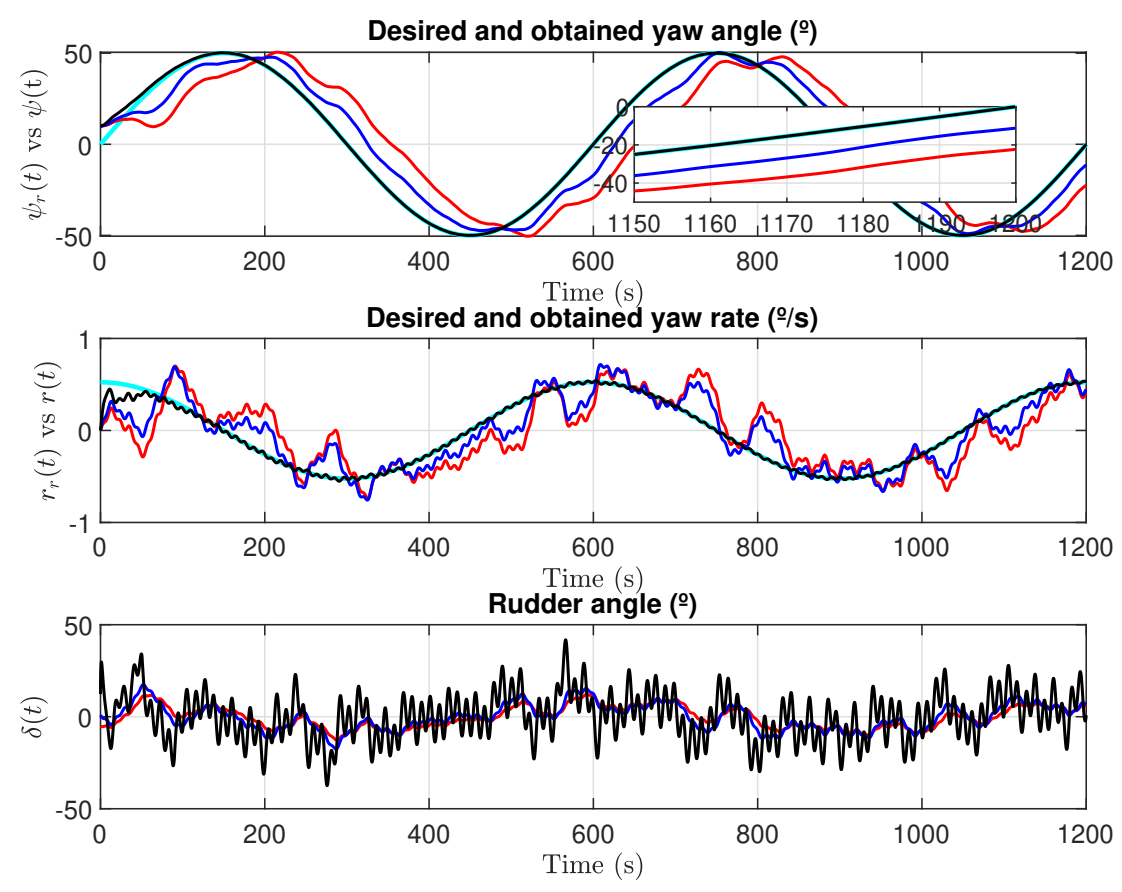

Figure 8. Sinusoidal yaw reference test with $d(t) \neq 0$. States and control.

vehicles. The solution has been proposed based on the application of adaptive gains that changes the dumping properties of the sliding surfaces and derives in a low/high gain profile such that it allows to overcome the use of large control inputs at initial conditions, keeping a desired higher gain at steady state.

The results obtained in the numerical simulations shows that the proposed AISM algorithm achieves the desired performance with fixed and time varying references cancelling the effect of the external disturbances. The performance is evaluated with a fixed parameter configuration that can be obtained from a settling time, maximum allowable yaw rate and steady state precision. The algorithm achieves the desire response without the need of develop a new parameter configuration for each type of test, showing its robustness properties.

An advantage of the method is its robustness with respect to an overestimation of $\dot{d}_{\text {max }}$ : the performance is not highly degraded if this bound is not accurately known. However, choosing an overly large value might cause oscillations in the response of the estimation error.

Another important aspect about the presented solution is its practical approach, such that a desired precision value of the yaw error at steady state is integrated in the design procedure in order to configure control parameters.

A deepest research of the functions that can be used to define the adaptive values of $\lambda(e)$ and $\delta(e)$ is an interesting open problem that can be analysed from the perspective of model predictive control in order to integrate an optimal point of view in the design of the adaptive parameters.

The extension of this procedure with the assumption of partial state feedback will be addressed in future researches by means of the application of an adaptive integral sliding mode observers.

Author Contributions: J. A. González-Prieto: Conceptualization, Methodology, Software, Formal analysis, Writing - original draft, C. Pérez-Collazo: Formal analysis, Writing - original draft, Y. Singh: Formal analysis, Writing - original draft.All authors have read and agreed to the published version of the manuscript. 
Acknowledgments: J. A. González-Prieto and C. Pérez-Collazo acknowledges funding from the Defense University Center at the Spanish Naval Academy, Spanish Ministry of Defense.

Y. Singh acknowledges the support of Industrial Systems Engineering (ISyE) Research Group at the Faculty of Engineering and Architecture, Ghent University and FlandersMake.

Conflicts of Interest: The authors declare that they have no known competing financial interests or personal relationships that could have appeared to influence the work reported in this paper..

\author{
Abbreviations \\ The following abbreviations are used in this manuscript: \\ USV Unmanned Surface Vehicle \\ IMO International Maritime Organisation \\ LOS Line of Sight \\ SMC Sliding Mode Control \\ AISM Adaptive Integral Sliding Mode \\ GUAS globally uniformly asymptotically stable \\ MAE Mean Absolute Error \\ MIA Mean Integral Absolute \\ MTV Mean Total Variation
}

\title{
References
}

1. Fossen, Thor I, "Handbook of marine craft hydrodynamics and motion control" John Wiley $\mathcal{E}$ Sons, 2011.

2. Do, Khac Duc and Pan, Jie, "Control of ships and underwater vehicles: design for underactuated and nonlinear marine systems" Springer Science E Business Media, 2009.

3. Liu, Tao and Dong, Zaopeng and Du, Hongwang and Song, Lifei and Mao, Yunsheng, "Path following control of the underactuated USV based on the improved line-of-sight guidance algorithm" Polish Maritime Research, 2017.

4. Liu, Zhiquan, "Ship adaptive course keeping control with nonlinear disturbance observer" IEEE Access, vol. 5, no. 16, pp. 17567-17575, 2017.

5. Ejaz, Muhammad and Chen, Mou, "Sliding mode control design of a ship steering autopilot with input saturation" International Journal of Advanced Robotic Systems, vol. 14, no. 3, pp. 1729881417703568, 2017.

6. Wan, Lili and Su, Yixin and Zhang, Huajun and Tang, Yongchuan and Shi, Binghua, "Global fast terminal sliding mode control based on radial basis function neural network for course keeping of unmanned surface vehicle" International Journal of Advanced Robotic Systems, vol. 16, no. 16, pp. 1729881419829961, 2019.

7. Rodriguez, Jonathan and Castañeda, Herman and Gordillo, JL, "Design of an adaptive sliding mode control for a micro-AUV subject to water currents and parametric uncertainties" Journal of Marine Science and Engineering, vol. 7, no. 12, pp. 445, 2019.

8. Zhang, Zhanshuo and Zhao, Yuhan and Zhao, Guang and Wang, Hongbo and Zhao, Y, "PathFollowing Control Method for Surface Ships Based on a New Guidance Algorithm" Journal of Marine Science and Engineering, vol. 9, no. 2, pp. 166, 2021.

9. Khaled, Nassim and Chalhoub, Nabil G, "A self-tuning guidance and control system for marine surface vessels" Nonlinear Dynamics, vol. 73, no. 1, pp. 897-906, 2013.

10. Budak, G and Beji, S, "Controlled course-keeping simulations of a ship under external disturbances" Ocean Engineering, vol. 218, pp. 108126, 2020.

11. Zhang, Xian-Ku and Zhang, Qiang and Ren, Hong-Xiang and Yang, Guang-Ping, "Linear reduction of backstepping algorithm based on nonlinear decoration for ship course-keeping control system" Ocean Engineering, vol. 147, pp. 1-8, 2018.

12. Islam, Muhammad Muzammal and Siffat, Syed Ahmad and Ahmad, Iftikhar and Liaquat, Muwahida, "Robust integral backstepping and terminal synergetic control of course keeping for ships" Ocean Engineering, vol. 221, pp. 108532, 2021.

13. Zhang, Xian-ku and Han, Xu and Guan, Wei and Zhang, Guo-qing, "Improvement of integrator backstepping control for ships with concise robust control and nonlinear decoration" Ocean Engineering, vol. 189, pp. 106349, 2019.

14. Zhang, Qiang and Zhang, Xianku, "Nonlinear improved concise backstepping control of course keeping for ships" Ieee Access, vol. 7, pp. 19258-19265, 2019. 
15. Zheng, Zewei and Sun, Liang, "Path following control for marine surface vessel with uncertainties and input saturation" Neurocomputing, vol. 177, pp. 158-167, 2016.

16. Zhang, Xian-ku and Zhang, Guo-qing, "Design of ship course-keeping autopilot using a sine function-based nonlinear feedback technique" The Journal of Navigation, vol. 69, no. 2, pp. 246-256, 2016.

17. Zhao, Hongbiao and Zhang, Xianku and Han, $\mathrm{Xu}$, "Nonlinear control algorithms for efficiencyimproved course keeping of large tankers under heavy sea state conditions" Ocean Engineering, vol. 189, pp. 106371, 2019.

18. Zhang, Qiang and Zhang, Xian-ku and Im, Nam-kyun, "Ship nonlinear-feedback course keeping algorithm based on MMG model driven by bipolar sigmoid function for berthing" International Journal of Naval Architecture and Ocean Engineering, vol. 9, no. 5, pp. 525-536, 2017.

19. Zhang, Qiang and Zhang, Xian-ku and Im, Nam-kyun, "Adaptive neural path-following control for underactuated ships in fields of marine practice" Ocean Engineering, vol. 104, pp. 558-567, 2015.

20. Zhang, Jun and Sun, Tairen and Liu, Zhilin, "Robust model predictive control for pathfollowing of underactuated surface vessels with roll constraints" Ocean Engineering, vol. 143, pp. 125-132, 2017.

21. Sharma, Sanjay K and Sutton, Robert and Motwani, Amit and Annamalai, Andy, "Non-linear control algorithms for an unmanned surface vehicle" Proceedings of the Institution of mechanical engineers, Part M: Journal of Engineering for the Maritime Environment, vol. 228, no. 2, pp. 146-155, 2014.

22. Yan, Zhaokun and Zhang, Xianku and Zhu, Huiying and Li, Zheng, "Course-keeping control for ships with nonlinear feedback and zero-order holder component" Ocean Engineering, vol. 209 , pp. 107461, 2020.

23. V. Utkin, J. Guldner and J. Shi, “Sliding Mode Control In Electro-mechanical Systems”, CRC press, 2009.

24. C. Edwards and S. Spurgeon, "Sliding Mode Control: Theory And Applications", CRC press, 1998.

25. A. Levant, "Robust exact differentiation via sliding mode technique" Automatica, vol. 34, no. 3, pp. 379-384, 1998.

26. C. Edwards, S. K. Spurgeon and R. J. Patton, "Sliding mode observers for fault detection and isolation" Automatica, vol. 36, no. 4, pp. 541-553, 2000.

27. Y. Feng, Y. Xinghuo and M. Zhihong, "Non-singular terminal sliding mode control of rigid manipulators" Automatica, vol. 38, no. 12, pp. 2159-2167, 2002.

28. S. G. Nersesov, H. Ashrafiuon and P. Ghorbanian, "On estimation of the domain of attraction for sliding mode control of underactuated nonlinear systems" International Journal of Robust and Nonlinear Control, vol. 24, no. 5, pp. 811-824, 2014.

29. Y. Hao, J. Yi, D. Zhao and D. Qian, "Robust control using incremental sliding mode for underactuated systems with mismatched uncertainties" American Control Conference, IEEE, 2008, pp. 532-537, 2008.

30. J. Liu, S. Laghrouche, M. Harmouche and M. Wack, “Adaptive-gain second-order sliding mode observer design for switching power converters" Control Engineering Practice, vol. 30, pp. 124-131, 2014

31. J. Yang, S. Li and X. Yu, "Sliding-mode control for systems with mismatched uncertainties via a disturbance observer" IEEE Transactions on Industrial Electronics, vol. 60, no. 1, pp. 160-169, 2013.

32. T. R. Oliveira, J. P. VS Cunha and L. Hsu, “Adaptive sliding mode control for disturbances with unknown bounds" 14th International Workshop on Variable Structure Systems (VSS). IEEE, 2016, pp. 59-64, 2016.

33. L. Hsu, T. R. Oliveira, J. P. VS Cunha and L. Yan, "Adaptive unit vector control of multivariable systems using monitoring functions" International Journal of Robust and Nonlinear Control, vol. 29, no. 3, pp. 583-600, 2019.

34. M. Chen, Q. Wu and R. Cui, "Terminal sliding mode tracking control for a class of SISO uncertain nonlinear systems" ISA transactions, vol. 52, no. 2, pp. 198-206, 2013.

35. W. Wang, X. D. Liu and J. Q. Yi, "Structure design of two types of sliding-mode controllers for a class of under-actuated mechanical systems" IET Control Theory \& Applications, vol. 1, no. 1, pp. 16388-172, 2007.

36. C. Edwards and Y. B. Shtessel, "Adaptive continuous higher order sliding mode control" Automatica, vol. 65, pp. 183-190, 2016. 
37. Feng, Yong and Han, Fengling and Yu, Xinghuo. Chattering free full-order sliding-mode control. Automatica, 4, vol. 50, pp. 1310-1314, 2014.

38. B. Bandyopadhyay, F. Deepak and K. Kim, "Sliding Mode Control Using Novel Sliding Surfaces", Springer, vol. 392, 2009.

39. González, José Antonio and Barreiro, Antonio and Dormido, Sebastián and Banos, Alfonso, "Nonlinear adaptive sliding mode control with fast non-overshooting responses and chattering avoidance", Journal of the Franklin Institute, vol. 354, no. 7, pp. 2788-2815, 2017.

40. J. A. González, A. Barreiro and S. Dormido "A practical approach to adaptive sliding mode control", International Journal of Control, vol. 17, no. 10, pp. 2452-2461, 2019.

41. Polyakov, Andrey "Generalized homogeneity in systems and control", Springer, 2020.

42. Mojallizadeh, Mohammad Rasool and Brogliato, Bernard and Acary, Vincent "Timediscretizations of differentiators: Design of implicit algorithms and comparative analysis", International Journal of Robust and Nonlinear Control, 2021.

43. Witkowska, Anna and Śmierzchalski, Roman, "Designing a ship course controller by applying the adaptive backstepping method", International Journal of Applied Mathematics and Computer Science, vol. 22, pp. 985-997, 2012.

44. Singh, Yogang and Sharma, Sanjay and Sutton, Robert and Hatton, Daniel, "Path planning of an autonomous surface vehicle based on artificial potential fields in a real time marine environment" Cardiff, 2017.

45. Polvara, Riccardo and Sharma, Sanjay and Wan, Jian and Manning, Andrew and Sutton, Robert, "Obstacle avoidance approaches for autonomous navigation of unmanned surface vehicles" The Journal of Navigation, vol. 71, no. 1, pp. 241-256, 2018.

46. Singh, Yogang and Sharma, S and Sutton, R and Hatton, D, "Optimal path planning of an unmanned surface vehicle in a real-time marine environment using a dijkstra algorithm" Marine Navigation. CRC Press, pp. 399-402 241-256, 2017.

47. Singh, Yogang and Sharma, Sanjay and Sutton, Robert and Hatton, Daniel and Khan, Asiya, "A constrained A* approach towards optimal path planning for an unmanned surface vehicle in a maritime environment containing dynamic obstacles and ocean currents" Ocean Engineering, vol. 169, pp. 187-201, 2018.

48. Panteley, Elena and Loria, Antonio, "On global uniform asymptotic stability of nonlinear time-varying systems in cascade", Systems \& Control Letters, vol. 33, no. 2, pp. 131-138, 1998. 